\title{
Correction for linear and non-linear distortions of STEM images
}

Pavel Potapov ${ }^{1}$ and Axel Lubk ${ }^{2}$

${ }^{1}$ IFW-Dresden, Dresden, Sachsen, Germany, ${ }^{2}$ IFW Dresden, Germany, United States

Acquisition of STEM images typically requires an order of magnitude longer time than recording broad beam TEM images. Since different instabilities always exist in the system, this might lead to notable distortions in the image. It is not exceptional to observe a slightly elliptic shape of the actually round particles or a monoclinic distortion of an otherwise rectangular crystal lattice. These distortions can be, however, corrected a posteriori. Since the STEM distortions depend on the scanning direction, the issue can be relaxed by taking two STEM images of the same area with mutually perpendicular scanning directions [1-3]. Analyzing such a couple of images allows to evaluate the STEM distortions and remove them by image warping. Fig. 1a shows the developed software tool for handling and correction of STEM images [4]. The correction is performed in two steps. First, the direction and magnitude of a linear drift in the course of acquisition is estimated either by the cross correlation or by the analysis of FFT transforms. Both images are corrected using affine transformations. In case of medium-magnification images, such linear correction might be sufficient to recover the true morphology of microstructures or the original shape of particles as illustrated in Fig. 1b.

Atomic-resolution images typically require to take into account non-linear distortions like a sudden drift of the sample, inaccuracy or instability of the scan generator and so on (Fig.2). The reconstruction relies on the assumption that there is no distortion within any given scanned row but distortions show up as the $\mathrm{X}, \mathrm{Y}$-shifts of the rows relative their "true" position [3]. The present work follows conceptually the approach [3] although technically uses a rather different algorithm. Namely, both images are sub-divided on a number of thin stripes and the cross-correlation between the stripes of differently oriented images is calculated. The continuous curves of the X,Y-shifts are then interpolated by cubic splines. As there is no fixed reference (both images are distorted), the procedure requires several iterations when one image uses another as a reference and other way around.

Acknowledgement:

The authors appreciate support from ERC (grant 715620 under the Horizon 2020 program) and DFG project 431448015. 
a

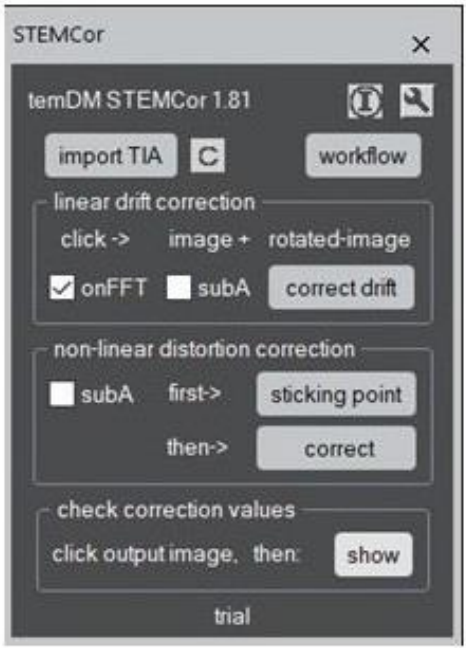

b
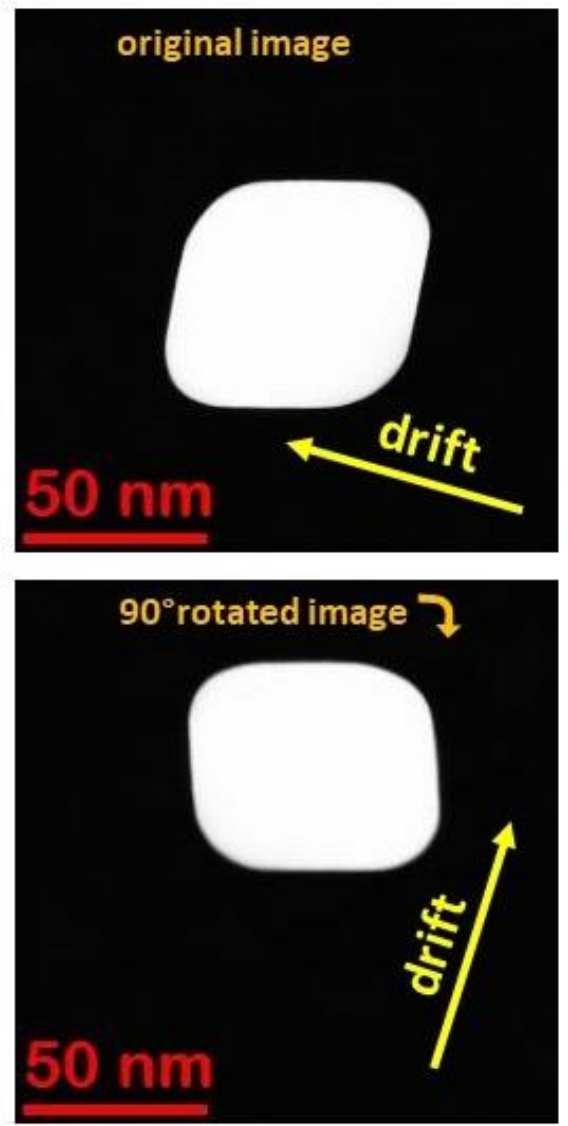
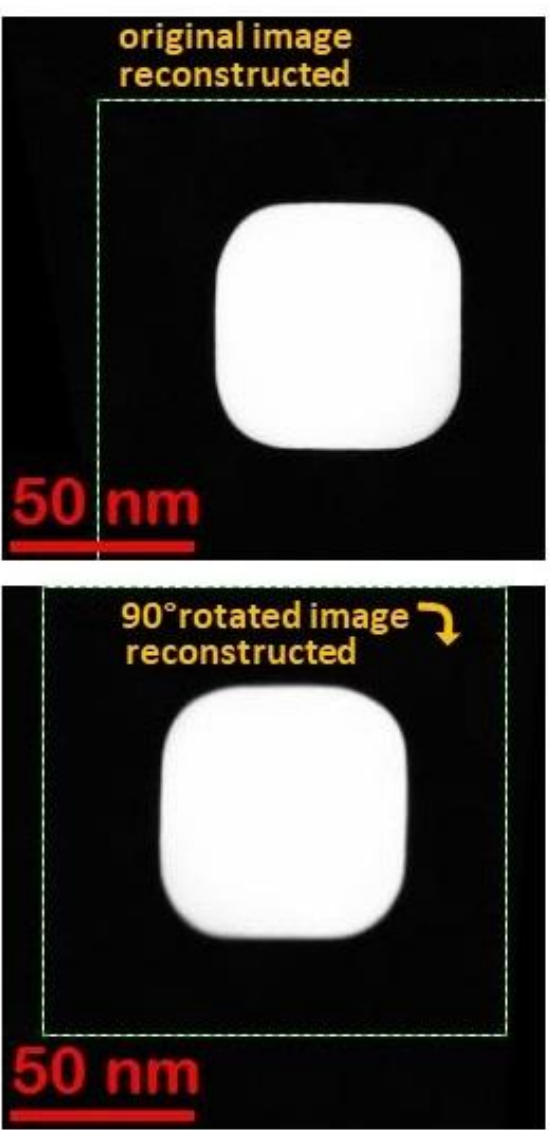

Figure 1. (a) Software tool for linear and non-linear STEM correction. (b) Linear correction of STEM images at medium magnification. The left couple of images shows an Au nanocube distorted in two mutually perpendicular directions due to a strong spatial drift. The corresponding right couple of images is corrected for the linear drift.

\section{after linear correction}

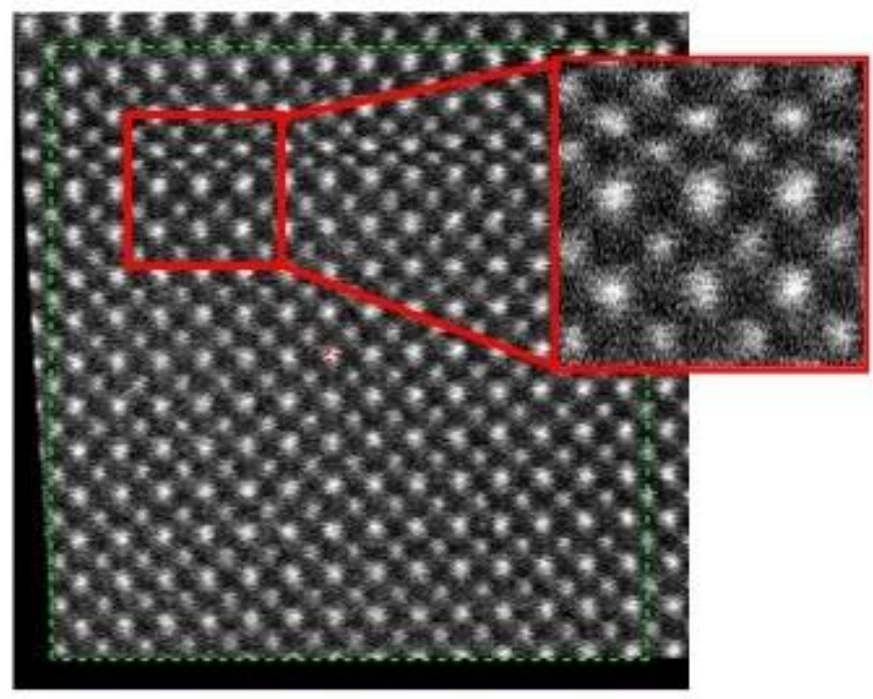

after non-linear correction

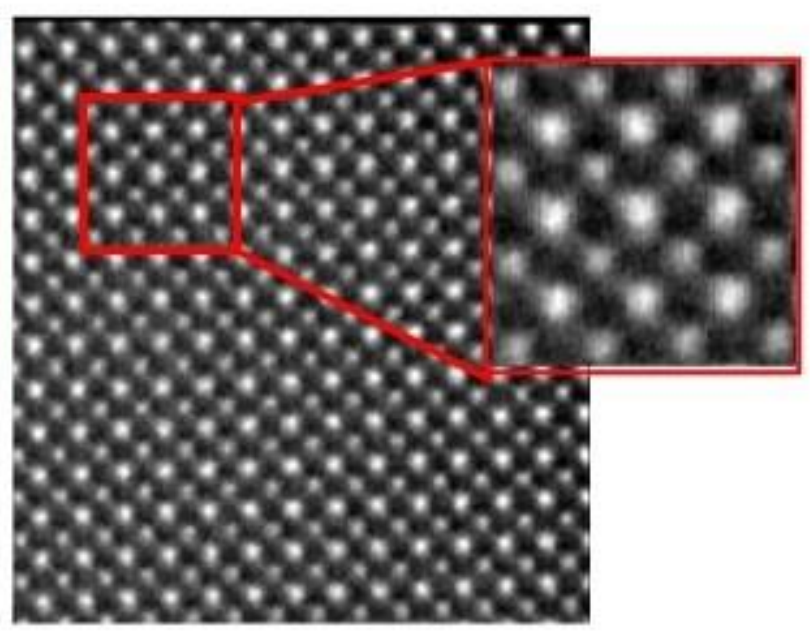


Figure 2. Non-linear correction of atomic-resolution STEM images of SrTiO3. The left image shows numerous distortions, e.g., the pronounced sudden jump between the scanning lines. The right image is corrected for the distortions, which improves the image quality and delivers the reliable information about the geometry of the lattice.

References

[1] L. Jones et al. Microscopy and Microanalysis 19 (2013) 1050.

[2] X. Sang et al. Ultramicroscopy 138 (2014) 28.

[3] C. Ophus et al. Ultramicroscopy162 (2016) 1.

[4] http://temdm.com/web/stem-correction/ 